\title{
The uplift of the Qinghai-Tibet Plateau: The driver behind the scene
}

Ruohan Zheng ${ }^{2}$, Wentao Jin ${ }^{3}$, Ting Zeng ${ }^{2,4}$, Xiaoyong Lu ${ }^{5}$, Haili Ran ${ }^{5}$, Yuhan Yang ${ }^{1}$, Qiuyun $\mathrm{Liu}^{1 *}$

${ }^{1}$ School of Life Sciences, Sun Yat-sen University, Guangzhou 510275, China.

${ }^{2}$ School of Chemistry, Sun Yat-sen University, Guangzhou 510275, China

${ }^{3}$ School of Medicine, Sun Yat-sen University, Guangzhou 510275, China

${ }^{4}$ College of Chemistry and Chemical Engineering, Jishou University, Jishou 416000, China

${ }^{5}$ School of Marine Sciences, Sun Yat-sen University, Guangzhou 510275, China.

Ruohan Zheng, Wentao Jin, Ting Zeng, Xiaoyong Lu, Haili Ran and Yuhan Yang made equal contributions to this work.

${ }^{*}$ Corresponding author: Qiuyun Liu, Ph.D, School of Life Sciences, Sun Yat-sen University, Guangzhou 510275, China. Tel: [+86 (020) 84110296]; E-mail: 1sslqy@mail.sysu.edu.cn 


\begin{abstract}
:
During the summer months, the northern hemisphere is tilted to the sun, and the Tibetan plateau and the Indian subcontinent are subjected to the solar pull. It is apparent that the upward component where the Tibetan plateau is subjected to is larger than that of the Indian subcontinent. In contrast, the horizontal component of the solar pull to the Indian subcontinent is larger than that of the Tibetan plateau. Additionally, large masses decelerate more slowly than smaller masses. Collectively these forces propel the Qinghai-Tibet Plateau upward and northeastward.
\end{abstract}


The Qinghai-Tibet Plateau is rising, albeit at minute rate. It is also moving northeastwardly at a tiny speed. ${ }^{1}$ What is behind such geophysical phenomena which shape the natural wonders of the planet? The sun, the earth and the moon are involved in an intricate trio dance ${ }^{2}$, and gravitational pulls hold these heavenly bodies together. Our earth, is rotating on a daily basis, in the solar and lunar gravitational fields. ${ }^{3}$ During the summer months, the northern hemisphere is tilted to the sun, and the Tibetan plateau and the Indian subcontinent are subjected to the solar pull if we put lunar pull aside to make things simpler. When we divide the solar pull into horizontal and vertical components, it is evident that the upward (vertical component) where the Tibetan plateau is subjected to is larger than that of the Indian subcontinent given equal amount of mass. In contrast, the horizontal component of the solar pull onto the Indian subcontinent is larger than that of the Tibetan plateau. Furthermore, according to Newton's Law of Inertia, large mass decelerates more slowly than smaller mass. ${ }^{4-5}$ Acting together, these forces push the Qinghai-Tibet Plateau upward and northward. During the winter months, the southern hemisphere is tilted to the sun. However the Indian Ocean to the south of India is moderate in depth, which do not drive a substantial southward drifting. Given the self-rotation of the earth, the Qinghai-Tibet Plateau is also drifting eastwardly. Taken together, the Qinghai-Tibet Plateau is rising slowly and moving northeastwardly. ${ }^{6}$

The Ocean depth to the north of Australia is about 5000 to 6000 meters, the fast northward acceleration of the pacific plate underneath ${ }^{5-10}$ during the summer months of the northern hemisphere drives Australia northward. Therefore, the gravitational pulls from the sun and the moon shape the landscape in a grand fashion.

\section{Acknowledgements}

Q.L. was supported by grants from the Science and Technology Transformation Program of Sun Yat-sen University of China (33000-18843234), Guangzhou Science and Technology Program (201804010328) and Guangdong Science and Technology Program (2016B020204001). We are grateful to Yan Shi for editing.

\section{References}

1. Guo J, Han W, Li X. The Cenozoic Tectonic Evolution of the West Qinling: Constraints on the Uplift and Deformation of the Qinghai-Tibet Plateau. Earth Science Frontiers 2009; 16(6):215-225

2. Yan S, Li J, Liu Q. Earthquakes-an intricate trio dance of gravitation. Science 2018; http://science.sciencemag.org/content/354/6315/1027/tab-e-letters.

3. Xu H, Wu J, Liu Q. Earth's self-rotation: high-velocity movements manifested in the solar and lunar gravitational fields. Science 2019; https://science.sciencemag.org/content/354/6308/88/tab-e-letters.

4. He R, He S, Nuertai X, et al. The faster acceleration of smaller masses in the west of Americas. Science 2017; http://science.sciencemag.org/content/354/6315/1027/tab-e-letters.

5. Liu Q, Gao J, Pan W, et al. Differentia in decelerations of masses: when the Earth 
meets lunar and solar pulls. Science 2017; http://science.sciencemag.org/content/354/6315/1027/tab-e-letters.

6. Zhu X, Yang F, Li Z, Liu Q. Why the Indian subcontinent drifts toward Eurasia. Science, 2017; http://science.sciencemag.org/content/356/6340/841/tab-e-letters

7. Wan Y, Shi Y, Li Z, et al. How focal depth is generated? Science 2017; http://science.sciencemag.org/content/354/6315/1027/tab-e-letters.

8. Yan S, Wang X, Qi J, Liu Q. Volcanoes or earthquakes: Wrist wrestling. Science, 2019; http://science.sciencemag.org/content/358/6370/1520/tab-e-letters.

9. Yan $\mathrm{S}, \mathrm{Xu} \mathrm{X}$, Chen L, et al. Earthquakes: The rocking cradle. Science 2019; https://science.sciencemag.org/content/354/6315/1027/tab-e-letters.

10. Zhong H, Wang K, Shi Y, et al. Tsunamis is a prime example of the vertical component of the forces during earthquakes. Science 2017; http://science.sciencemag.org/content/358/6367/1164/tab-e-letters. 